\title{
UNSTABLE VIBRATIONS AND BUCKLING OF ROTATING FLEXIBLE RODS*
}

\author{
$\mathrm{BY}$ \\ W. D. LAKIN (University of Toronto) \\ AND \\ A. NACHMAN (Texas A\&M University)
}

\begin{abstract}
We consider a group of fourth-order boundary-value problems associated with the small vibrations or buckling of a uniform flexible rod which is clamped at one end and rotates in a plane perpendicular to the axis of rotation. The vibrations may be in any plane relative to the plane of rotation and the rod is off-clamped, i.e. the axis of rotation does not pass through the rod's clamped end. The governing equation for the vibrations involves a small parameter for rapid rotation and must be treated by singular perturbation methods. Further, a turning point of the equation always coincides with a boundary point. Both free and unstable vibrations are examined, and a stability boundary is obtained. Results for the unstable vibrations predict the unexpected existence of a time-independent buckled state in non-transverse planes when the rod is wholly under tension. The general buckling problem in the transverse plane is also considered.
\end{abstract}

1. Introduction. This work considers several boundary-value problems arising in connection with the small-amplitude vibration or buckling of flexible rods which are clamped at one end and rotate with constant angular velocity in a plane perpendicular to the axis of rotation. The rod is assumed not to twist, and to have uniform density and cross-section. Eigenvalues of the boundary-value problems determine either the rod's natural frequencies of vibration, the growth rate for unstable vibrations, or the critical rotation rate for buckling.

Recent interest in rotating flexible rods stems from applications to helicopter rotor blades and spin-stabilized space satellites. Both of these cases are axis-clamped, i.e. the axis of rotation passes through the clamped end of the rod. The free transverse vibrations of axis-clamped rods have been studied previously by Boyce, Handleman, and DiPrima [1], Lakin [5], and Lakin and $\mathrm{Ng}$ [7].

Vibrations of axis-clamped rods are now relatively well understood. In the case of a wobbling rotor, however, the axis of rotation does not pass through the rod's clamped end. Indeed, as the rod turns, its clamped end describes a circle of radius $\mathrm{R}>0$ about the axis of rotation. This has prompted Lakin [6] to study the transverse vibrations of flexible rods clamped off the axis of rotation. If the rod has length $\mathrm{L}$, the physical configuration in this off-clamped case is determined by the dimensionless ratio $\alpha=\mathrm{R} / \mathrm{L}$. Small but positive

\footnotetext{
* Received September 28, 1976; revised version received March 17, 1977. This work has been supported in part by the National Research Council of Canada under grant A7850 (W.D.L) and a College of Science Research Grant. Texas A \& M University (A.N.).
} 
$\alpha$ corresponds to the wobbling axis-clamped rod. For $\alpha>1$, the situation is similar to a rod which is clamped to the rim of a rotating wheel and extends inward toward the axis of rotation like a partial spoke. This situation is shown in Fig. 1.

In the present paper, we consider rapidly rotating rods with $\alpha \geq 0$ which can vibrate in a plane making an arbitrary angle $\theta$ with the plane of rotation. The relationship between the planes of rotation and vibration is illustrated in Fig. 2. Small transverse vibrations $(\theta=$ $\pi / 2$ ) are known to be stable for $0 \leq \alpha<\frac{1}{2}$. However, we show that small vibrations in nontransverse planes may become unstable for $\alpha$ in this range. Indeed, vibrations in the plane of rotation $(\theta=0)$ are found to be unstable for even relatively small values of $\alpha$. Results for fixed off-clamping $\alpha$ and variable angle $\theta$ lead to a stability boundary for the free vibrations in the $(\alpha, \theta)$-plane. In addition, information on the unstable vibrations predicts the existence of a single time-independent buckled mode in non-transverse planes when $\alpha$ $<\frac{1}{2}$. Buckling is unexpected in this case as the rod is wholly under tension. We also investigate the buckling problem in the transverse plane when $\alpha>\frac{1}{2}$. Buckling in the plane of rotation has been previously discussed by Nachman [11] and Mostaghel and Tadjbakhsh [10].

2. Boundary-value problem for vibrations. To treat the present vibration problem, let $s$ denote arc length along the rod measured from the clamped end $s=0$, and let $u(s, t)$ be the displacement of the rod in the plane of vibration where the angle between the planes of rotation and vibration is $\theta$ and $0 \leq \theta \leq \pi / 2$. The amplitude of the vibrations will be assumed sufficiently small that the governing equation may be consistently linearized. If the rod has length $L$, cross-sectional area $A$, mass per unit volume $\rho$, bending stiffness $E I$, and rotates with constant angular velocity $\Omega$, the partial differential equation for the small vibrations can be written as

$$
E I \frac{\partial^{4} u}{\partial s^{4}}-\frac{\partial}{\partial s}\left\{P(s) \frac{\partial u}{\partial s}\right\}=-\rho A\left\{\frac{\partial^{2} u}{\partial t^{2}}-\Omega^{2}\left(\cos ^{2} \theta\right) u\right\},
$$

where

$$
P(s)=\rho A \Omega^{2}(L-s)\left[\frac{1}{2}(L+s)-R\right] .
$$

At the clamped end of the rod $(s=0)$, we must have zero displacement and slope, while at the free end $(s=L)$ both shear force and bending moment must vanish. Hence

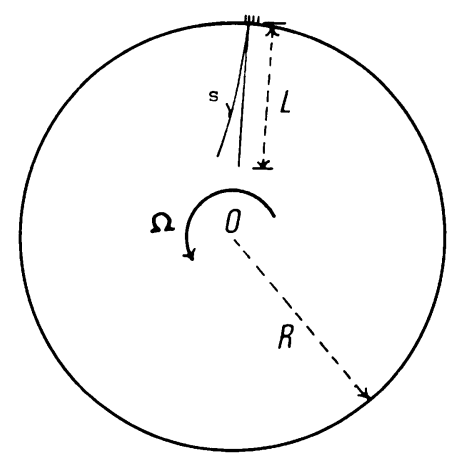

FIG. 1. Flexible rod fixed to the rim of a rotating wheel. 


$$
\begin{aligned}
u(0, t) & =\partial u / \partial s(0, t)=0, \\
\frac{\partial^{2} u}{\partial s^{2}}(L, t) & =\frac{\partial^{3} u}{\partial s^{3}}(L, t)=0 .
\end{aligned}
$$

We will seek harmonic vibrations of the form $u(s, t)=w(s) \exp (i \omega t)$. The system $(2.1-3)$ may now be put in a form more suitable for mathematical analysis by introducing the dimensionless variable $x=-s / L$ and the dimensionless ratios

$$
\tilde{\epsilon}^{3}=E I / \rho A \Omega^{2} L^{4}, \quad \alpha=R / L, \quad \lambda=(\omega / \Omega)^{2} .
$$

With these scalings, the governing equation becomes

$$
\tilde{\epsilon}^{3} w^{\text {iv }}-\frac{1}{2}(1+x)(1-2 \alpha-x) w^{\prime \prime}+(x+\alpha) w^{\prime}-\mu w=0,
$$

where the eigenvalue $\mu$ is $\mu=\lambda+\cos ^{2} \theta$. Associated boundary conditions are

$$
w(0)=w^{\prime}(0)=0, \quad w^{\prime \prime}(-1)=w^{\prime \prime \prime}(-1)=0 .
$$

Throughout this work, we will assume rapid rotation so the parameter $\tilde{\epsilon}$ will be small, real and positive.

The distinctive character of the governing differential equation (2.5) comes from the two turning points at $x_{1}=-1$ and $x_{2}=1-2 \alpha$. The position of the second turning point $x_{2}$ will shift as $\alpha$ varies. However, independent of $\alpha$, in the boundary-value problem the turning point $x_{1}$ is always at an end point.

If, for fixed values of $\alpha$ and $\theta$, the boundary-value problem $(2.5,6)$ gives a value for the smallest eigenvalue $\mu_{0}$ such that $\lambda_{0}$ is positive, then the off-clamped rod can sustain free vibrations in a plane making an angle $\theta$ with the plane of rotation. In this case, the eigenvalues $\mu_{m}$ determine the natural frequencies of vibration. If, however, $\mu_{0}-\cos ^{2} \theta$ is negative, $\omega_{0}$ will have a square root with negative imaginary part. Hence, for that root $\exp \{i \omega t\}$ will have as its real part a growing exponential so the small vibrations will amplify with time, i.e., they will be unstable. Vibrations of the axis-clamped $\operatorname{rod}(\alpha=0)$ are known to be stable for all $\theta$. For off-clamped rods, the transverse vibrations $(\theta=\pi / 2)$ are known to be stable at least up to $\alpha<\frac{1}{2}$.

3. Non-transverse vibrations of off-clamped rotating rods. To bring out explicitly
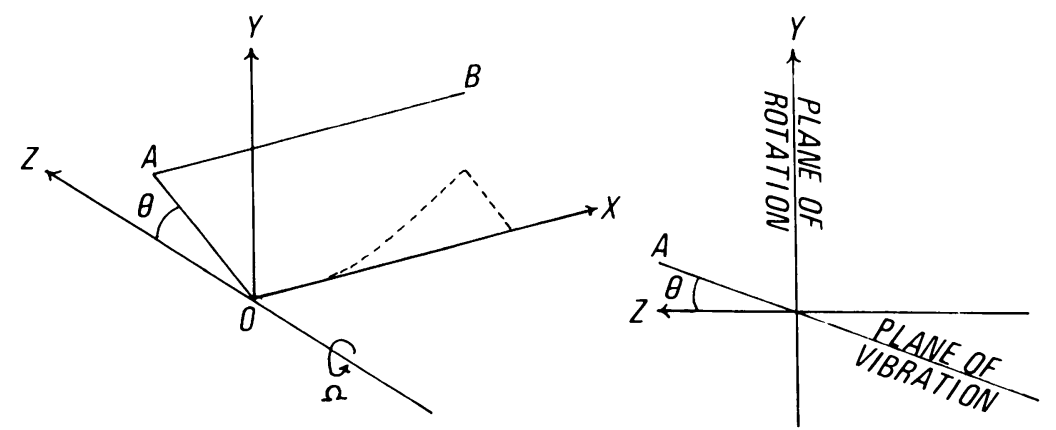

FIG. 2. Geometry for an axis-clamped rod which rotates in the XY-plane and vibrates in the plane OAB defined by the angle $\theta$. 
the dependence on $\alpha$ and $\theta$, let $\lambda$ be written as $\lambda(\alpha, \theta)$. Then, $\lambda(\alpha, \pi / 2) \equiv \mu$, and we have

$$
\lambda(\alpha, \theta)=\lambda(\alpha, \pi / 2)-\cos ^{2} \theta .
$$

Hence, information on the non-transverse vibrations for $\alpha \geq 0$ may be obtained from the transverse vibration case.

For axis-clamped rods, second approximations to $\lambda_{n}(0, \pi / 2)$ obtained in [7] for $n=0$, $1,2, \cdots$ are

$$
\lambda_{n}(0, \pi / 2)=(n+1)(2 n+1)+\tilde{\epsilon}^{32} \frac{4 n+3}{2^{4 n+1 / 2}} \frac{\{(2 n+1) !\}^{2}}{\{n !\}^{4}}+0\left(\tilde{\epsilon}^{3}\right) .
$$

Eq. (3.1) shows that $\lambda_{0}(0, \theta)$ is positive for all $\theta$, confirming that axis-clamped rods can sustain small free vibrations in any plane.

When $\alpha$ is small, positive, but larger than $O\left(\tilde{\epsilon}^{3 / 2}\right)$, Lakin [6] has found that the transverse eigenvalues are lowered by an $O(\alpha)$ amount. In particular,

$$
\lambda_{n}(\alpha, \pi / 2)=(n+1)(2 n+1)-\frac{2 \alpha(4 n+3)}{\pi(1-\alpha)}+0\left(\tilde{\epsilon}^{32}, \alpha^{2}\right) .
$$

Provided $\theta$ is not small, the non-transverse eigenvalue $\lambda_{0}(\alpha, \theta)$ remains positive. However, if $\theta$ is small, i.e. the planes of vibration and rotation nearly coincide, unstable situations with $\lambda_{0}$ negative are possible. In this case

$$
\lambda_{0}(\alpha, \theta)=\theta^{2}-6 \alpha / \pi+0\left(\tilde{\epsilon}^{3 / 2}, \alpha^{2}, \theta^{4}\right) .
$$

Vibrations in planes with angles close to $\theta=0$ will thus be unstable for even small offclamping.

For $0<\alpha<\frac{1}{2}$, it was shown in [6] that first approximations to the transverse eigenvalues come from zeros in degree of the Legendre function $P_{\nu}(\alpha /(\alpha-1))$. If the positive zeros are $\nu_{n}$, then $\lambda_{n}(\alpha, \pi / 2)=\nu_{n}\left(\nu_{n}+1\right) / 2$. The first few eigenvalues must be obtained numerically (see Fig. 3). Analytic expressions for the larger $\lambda_{n}$ may be obtained using the asymptotic expansion of $P_{\nu}$ for large $\nu$.

As $\alpha$ increases toward $\frac{1}{2}$, Fig. 3 shows that $\lambda_{0}(\alpha, \pi / 2)$ decreases monotonically toward zero. Conversely, as $\theta$ decreases toward $0, \cos ^{2} \theta$ increases monotonically toward one. Thus, from (3.1), for a fixed value of $\alpha$ there will be a single critical angle $\theta_{c}(\alpha)$ at which $\lambda_{0}(\alpha, \theta)$ vanishes identically. The eigenvalue $\lambda_{o}(\alpha, \theta)$ will be positive for $\theta>\theta_{c}$ but negative for $\theta<\theta_{c}$. Vibrations in planes with angles $\theta<\theta_{c}(\alpha)$ will now be unstable. The curve $\theta=$ $\theta_{c}(\alpha)$ plotted in Fig. 4 thus gives a stability boundary for the vibrations in the $(\alpha, \theta)$-plane. Eq. (3.1) also shows that the unstable vibration with the maximum growth rate will always be in the plane of rotation $\theta=0$. To determine critical off-clampings, the stability boundary may be written as $\alpha=\alpha_{c}(\theta)$. Vibrations in a plane with angle $\theta$ will now be unstable if $\alpha>\alpha_{c}$.

If $\alpha$ is close to $\frac{1}{2}$ with $\frac{1}{2}-\alpha$ positive and larger than $\mathrm{O}(\tilde{\epsilon})$, a formula of Hobson [4] may be used to give for $n=0,1,2, \cdots$

$$
\lambda_{n}(\alpha, \pi / 2) \sim \frac{n(n+1)}{2}+\frac{2 n+1}{2 \pi} \tan ^{-1}\left[\frac{\pi}{\log \left(\frac{1}{1-2 \alpha}\right)}\right] .
$$

This expression, which was not contained in [6], shows that $\lambda_{0}(\alpha, \pi / 2) \rightarrow 0$ as $\alpha \rightarrow 1 / 2-$. Hence, from (3.1), as $\alpha$ increases toward $1 / 2^{-}$, only vibrations in planes very close to 


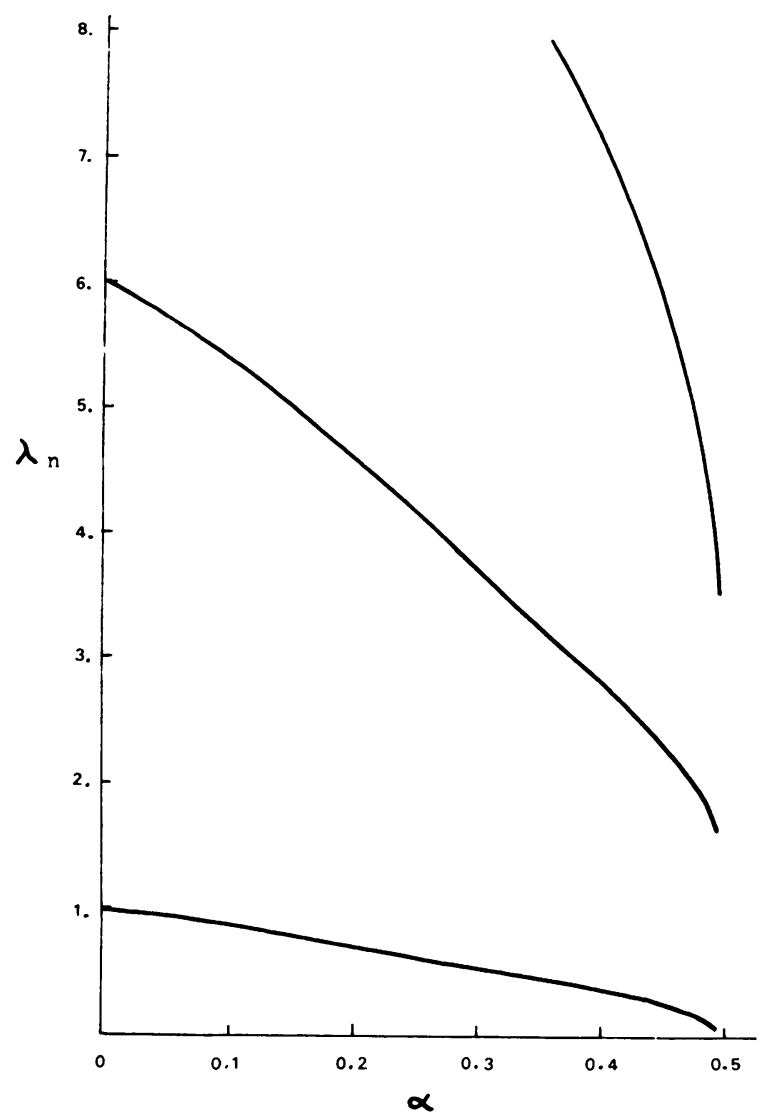

FIG. 3. The eigenvalues $\lambda_{n}(\alpha, \pi / 2)$ for the transverse vibrations.

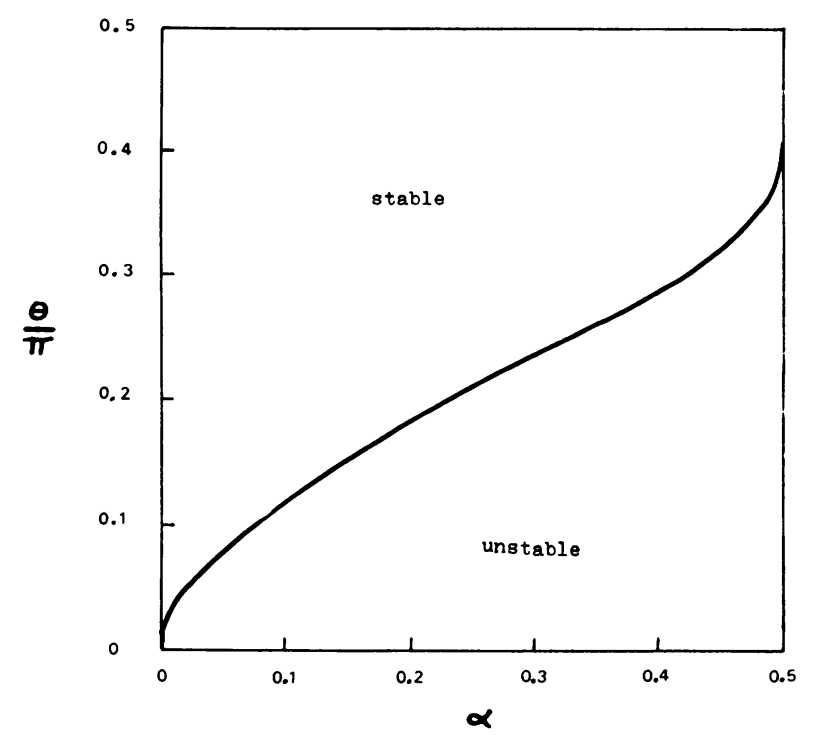

FIG. 4. The stability boundary in the $(\alpha, \theta)$-plane with $\theta$ scaled by $\pi$. 
transverse can remain stable. In light of these results, it seems likely that even transverse vibrations will not remain stable past $\alpha=1 / 2$.

4. Unstable transverse vibrations. In this section, we will examine the boundaryvalue problem $(2.5,6)$ for the transverse vibrations $\theta=\pi / 2$ when $\alpha>1$. Define new independent and dependent variables by the relations

$$
y=\frac{x+\alpha}{1-\alpha}, \quad \phi(y)=w(x) .
$$

Then Eq. (2.5) becomes

$$
\epsilon^{3} \phi^{\mathrm{iv}}-1 / 2\left(1-y^{2}\right) \phi^{\prime \prime}+y \phi^{\prime}-\lambda \phi=0,
$$

where $\epsilon^{3}=\tilde{\epsilon}^{3}(1-\alpha)^{-4}$ and $\lambda=\lambda(\alpha, \pi / 2)$. The associated boundary conditions (2.6) now become

$$
\begin{array}{r}
\phi\left(\frac{\alpha}{1-\alpha}\right)=\phi^{\prime}\left(\frac{\alpha}{1-\alpha}\right)=0, \\
\phi^{\prime \prime}(-1)=\phi^{\prime \prime \prime}(-1)=0 .
\end{array}
$$

Eq. (4.2) is associated with the transverse vibrations of an axis-clamped rod. However, the boundary point $y_{0}=\alpha /(1-\alpha)$ in the first two conditions in (4.3) is radically different from the corresponding boundary point in the axis-clamped problem. In particular, $y_{0}$ now lies to the left of the turning point $y_{1}=-1$ on the $y$-axis. Hence, all outer approximations to a set of linearly independent solutions of Eq. (4.2) will be balanced at $y_{0}$. In the eigenvalue relation, there will thus not be the mixture of exponentially large, balanced, and exponentially small terms which simplifies analysis when $0 \leq \alpha<1 / 2$.

The present work will use real-variable methods to obtain an asymptotic solution of the boundary-value problem $(4.2,3)$. Previous treatments of Eq. (4.2) for small $\epsilon$ have considered $y$ to be a complex variable. In particular, matched inner and outer approximations and Stokes multipliers for solutions of (4.2) in the complex $y$-plane were obtained in [5]. This earlier complex-variable approach was partially motivated by corresponding developments for the Orr-Sommerfeld problem of hydrodynamic stability. Indeed, the generalized Airy functions used in [5] were developed by Reid [13] in the stability context. For the Orr-Sommerfeld problem, the small parameter is complex, and hence solutions must be obtained in the complex plane. Uniform asymptotic approximations to solutions of the Orr-Sommerfeld equation have been derived by Reid [14]. In the present problem, however, $\epsilon$ is a real parameter and the turning point is also a boundary point. Under these conditions, it is not necessary to use complex variable methods. We may thus avoid complications arising from the Stokes phenomenon in the complex plane.

Preliminary transformation. To bring out explicitly the turning point nature of Eq. (4.2), define the real variable $\eta$ and the new dependent variable $\chi(\eta)$ by the relations

$$
\eta(y)=\frac{1}{2}\left\{3 \int_{-1}^{y}(1-y)^{1 / 2} d y\right\}^{2 / 3}, \chi(\eta)=\phi(y) .
$$

Eq. (4.2) now becomes

$$
\epsilon^{3}\left(\chi^{\mathrm{iv}}+6 \gamma \chi^{\prime \prime \prime}\right)-\left(\eta+\epsilon^{3} f_{1}(\eta)\right) \chi^{\prime \prime}-\left(h_{0}(\eta)+\epsilon^{3} h_{1}(\eta)\right) \chi^{\prime}-\lambda \eta^{-4} \chi=0
$$

where $h_{0}, h_{1}$, and $f_{1}$ are analytic functions of $\eta$ and 


$$
\gamma(\eta)=\eta^{\prime \prime}(y) /\left[\eta^{\prime}(y)\right]^{2}, h_{0}(\eta)=1+3 \gamma \eta .
$$

The turning point $y_{1}=-1$ now corresponds to $\eta_{1}=0$ and $\eta^{\prime}(-1)=1$. The second boundary point $y_{0}=\alpha /(1-\alpha)$ becomes

$$
\eta_{0}=\eta\left(y_{0}\right)=-\frac{1}{2}\left\{\frac{\alpha(2 \alpha-1)^{1 / 2}}{(\alpha-1)^{2}}-\log \left(\frac{\alpha+(2 \alpha-1)^{1 / 2}}{\alpha-1}\right)\right\} .
$$

Four linearly independent solutions of Eq. (4.5) may be defined to within multiplicative constants by their asymptotic properties away from the turning point. The two solutions $\chi_{1}(\eta)$ and $\chi_{2}(\eta)$ are balanced. However, $\chi_{2}$ behaves logarithmically away from $\eta$ $=0$. Care must be taken with $\chi_{2}$ as adding arbitrary multiples of $\chi_{1}$ will not change the defining asymptotic behavior. For positive $\eta$, the solutions $\chi_{3}(\eta)$ and $\chi_{4}(\eta)$ are recessive and dominant, respectively. These four solutions form a numerically satisfactory set in the sense of Miller [9].

Approximation functions and Wronskians. In the present boundary-value problem, for bounded non-positive $\eta$ we require uniformly valid approximations to the four solutions $\chi_{j}(\eta)$. Approximations to $\chi_{1}(\eta)$ will be power series in $\eta$. However, approximations to $\chi_{2}(\eta), \chi_{3}(\eta)$, and $\chi_{4}(\eta)$ will involve the Scorer function $H i(x)$ and the usual Airy functions $A i(x)$ and $B i(x)$ together with first integrals and derivatives of these functions. To standardize notation, let

$$
\begin{gathered}
A i(x, 0)=A i(x), \quad B i(x, 0)=B i(x), \quad H i(x, 0)=\pi H i(x), \\
A i(x,-1)=A i^{\prime}(x), \quad B i(x,-1)=B i^{\prime}(x), \quad H i(x,-1)=\pi H i^{\prime}(x), \\
A i(x, 1)=\int_{0}^{x} A i(t) d t-\frac{1}{3}, \\
B i(x, 1)=\int_{0}^{x} B i(t) d t \quad \text { and } H i(x, 1)=\pi \int_{0}^{x} H i(t) d t .
\end{gathered}
$$

Then, if $C i$ denotes $A i, B i$, or $H i$,

$$
\frac{d}{d x} C i(x, p)=C i(x, p-1) \quad(p=0,1)
$$

and

$$
\frac{d}{d x} \operatorname{Ci}(x,-1)=x \operatorname{Ci}(x, 0)+\delta i
$$

where $\delta i=1$ if $C i$ is $H i$ and $\delta i=0$ otherwise. Second-order Wronskians of these functions are

$$
\begin{aligned}
& W[A i(x, 0), B i(x, 0)]=1 / \pi \\
& W[A i(x, 1), B i(x, 1)]=\pi^{-1} H i(x, 0)-B i(x, 0), \\
& W[A i(x, 0), H i(x, 0)]=A i(x, 1)+1, \\
& W[B i(x, 0), H i(x, 0)]=B i(x, 1) .
\end{aligned}
$$

The Wronskian of $\operatorname{Hi}(x, 1)$ with either $A i(x, 1)$ or $B i(x, 1)$ is not needed in the present problem. However, we note that if $D=d / d x, A=D^{2}-x$, and $C i$ is either $A i$ or $B i$, 


$$
(A D-1) W[C i(x, 1), H i(x, 1)]=2 C i(x, 0) .
$$

Hence, these two Wronskians may be expressed in terms of real-variable analogues of generalized Airy functions.

Outer approximations. Before considering the uniform approximations themselves, we will briefly examine approximations valid away from $\eta=0$ for solutions of (4.5). Portions of these outer approximations will be required to describe the slowly varying coefficients in the uniform approximations.

Outer approximations to $\chi_{1}(\eta)$ and $\chi_{2}(\eta)$ may be obtained by expanding $\chi$ in $(4.5)$ in powers of $\epsilon^{3}$. First approximations $\bar{\chi}_{1}{ }^{10}$ and $\bar{\chi}_{2}{ }^{(0)}$ are now solutions of the reduced equation

$$
R_{2} \bar{\chi}^{0 \prime}=\left(\eta \frac{d^{2}}{d \eta^{2}}+h_{0}(\eta) \frac{d}{d \eta}+\lambda \eta^{\prime-4}\right) \bar{\chi}^{01}=0 \text {. }
$$

This equation may be solved by the method of Frobenius, giving

$$
\bar{\chi}_{1}{ }^{0}(\eta)=1-\lambda \eta+\frac{\lambda}{4}\left(\lambda-\frac{7}{5}\right) \eta^{2}+O\left(\eta^{3}\right)
$$

and

$$
\begin{gathered}
\bar{\chi}_{2}{ }^{0}(\eta)=P(\eta)+\bar{\chi}_{1}{ }^{0}(\eta) \log |\eta| \\
P(\eta)=-\psi(1)+\left[\frac{3}{5}-\lambda(\psi(2)+1)\right] \eta+O\left(\eta^{2}\right)
\end{gathered}
$$

where $\psi(x)=\Gamma^{\prime}(x) / \Gamma(x)$ is the digamma function. However, the reduced equation (4.13) is simply a transformed version of the reduced equation

$$
\frac{1}{2}\left(1-y^{2}\right) \frac{d^{2} \Phi^{0}}{d y^{2}}-y \frac{d \Phi^{0}}{d y}+\lambda_{\phi}^{(0)}=0
$$

corresponding to setting $\epsilon=0$ in Eq. (4.2), and solutions of this equation are linear combinations of the Legendre functions $P_{\nu}(-y)$ and $Q_{\nu}(-y)$ with $\nu(\nu+1)=2 \lambda$. For later use, we note that $\bar{\chi}_{1}{ }^{0 \prime}$ and $\bar{\chi}_{2}{ }^{0 \prime}$ may be written as

$$
\begin{gathered}
\bar{\chi}_{1}{ }^{0}(\eta)=P_{\nu}(-y), \\
\bar{\chi}_{2}{ }^{(0)}(\eta)=-2 Q_{\nu}(-y)+[\log 2+\psi(1)-2 \psi(\nu+1)] P_{\nu}(-y) .
\end{gathered}
$$

Outer approximations to $\chi_{3}(\eta)$ and $\chi_{4}(\eta)$ for positive $\eta$ may be obtained by the WKBJ technique. For $\bar{\chi}_{3}(\eta)$ we have

$$
\begin{gathered}
\bar{\chi}_{3}(\eta)=-\frac{1}{2} \pi^{-1 / 2} \epsilon^{3 / 4}\left(\eta \eta^{\prime 2}\right)^{-3 / 4} \exp \left(-\frac{2}{3} \epsilon^{-3 / 2} \eta^{3 / 2}\right)\left\{1-\epsilon^{3 / 2} G_{1}(\eta)+O\left(\epsilon^{3}\right)\right\} \\
G_{1}(\eta)=\frac{41}{48} \eta^{-3 / 2}-\left(\lambda+\frac{3}{10}\right) \eta^{-1 / 2}+\int_{0}^{\eta} G(\eta) d \eta \\
G(\eta)=-\frac{3}{4}\left(\gamma+\frac{1}{5}\right) \eta^{-3 / 2}+\frac{1}{4}\left(\frac{\gamma^{2}}{2}+7 \gamma^{\prime}\right) \eta^{-1 / 2}+1 / 2 \lambda\left(\eta^{\prime-4}-1\right) \eta^{-3 / 2}
\end{gathered}
$$

$G(\eta)$ is regular and $G_{1}(\eta)$ contains no constant term at $\eta=0$. The corresponding WKBJ approximation $\bar{\chi}_{4}$ will be exponentially large for positive $\eta$. Normalizations of these outer approximations have been chosen for later convenience.

We now wish to derive uniform approximations to the solutions of Eq. (4.5). 
The regular balanced solution. The regular solution $\bar{\chi}_{1}{ }^{i 0)}$ of the reduced equation (4.13) will provide a uniform first approximation to $\chi_{1}(\eta)$. We thus have

$$
\chi_{1}(\eta)=\bar{\chi}_{1}^{10}(\eta)+O\left(\epsilon^{3}\right) .
$$

The logarithmic balanced solution. To infer the structure of the uniform approximations to $\chi_{2}(\eta)$, we note that away from the turning point for negative $\eta$ this solution must behave like $\log |\eta|$. Further, the leading term of the inner expansion for $\chi_{2}$ is a solution of the equation

$$
D A D \tilde{\chi}_{2}^{(0)}(\zeta)=0
$$

where

$$
\zeta=\eta / \epsilon
$$

is a stretched variable and $D=d / d \zeta, A=D^{2}-\zeta$. A solution of (4.19) with appropriate asymptotic behavior is $\mathrm{Hi}(\zeta, 1)$. Second and third inner approximations will involve $\mathrm{Hi}(\zeta$, $0), H i(\zeta,-1)$, and polynomials in $\zeta$. This suggests that to order $\epsilon^{3}$ the uniform expansion for $\chi_{2}$ must be of the form

$$
\begin{gathered}
\chi_{2}(\eta)=\mathbf{G}(\eta)-\mathbf{A}(\eta) H i(\zeta, 1)-\epsilon^{2} \mathbf{B}(\eta) H i(\zeta, 0) \\
-\epsilon \mathbf{C}(\eta) H i(\zeta,-1)+O\left(\epsilon^{3}\right)
\end{gathered}
$$

where the slowly varying coefficients $\mathbf{A}, \mathbf{B}, \mathbf{C}$, and $\mathbf{G}$ do not depend on $\epsilon$ and are regular at $\eta=0$.

Differential equations for the four coefficients may be obtained by substituting (4.21) into Eq. (4.5) and requiring that the result vanish to lowest order. We have that with $R_{2}$ as in $(4.13)$,

$$
\begin{gathered}
R_{2} \mathrm{G}+4 \mathrm{~A}^{\prime}+6 \gamma \mathrm{A}+4 \eta \mathrm{C}^{\prime}+\left(3+6 \gamma \eta-h_{0}\right) \mathrm{C}=0, \\
R_{2} \mathrm{~A}=0,
\end{gathered}
$$

and

$$
\left(2 \eta \frac{d}{d \eta}+1+6 \gamma \eta-h_{0}\right)(\mathbf{A}+\eta \mathbf{C})=0 .
$$

The corresponding equation involving $\mathbf{B}$ is quite complicated, and an alternate way to determine this coefficient will be discussed shortly. However, the equations for $\mathbf{A}, \mathbf{C}$, and $\mathbf{G}$ are readily solvable. Eq. (4.23) implies that $\mathbf{A}$ must be a multiple of the regular solution of the reduced equation, and hence

$$
\boldsymbol{A}(\eta)=\mathrm{A}(0) \bar{\chi}_{1}{ }^{0}(\eta) .
$$

Similarly, Eq. (4.24) may be integrated immediately to give

$$
\boldsymbol{A}(\eta)+\eta \mathbf{C}(\eta)=\mathbf{A}(0) \eta^{\prime-3 / 2}
$$

Eliminating $\mathbf{A}$ and $\mathbf{C}$ from Eq. (4.22) now shows that

$$
R_{2} \mathrm{G}=-\mathbf{A}(0)\left\{2 \bar{\chi}_{1}{ }^{\prime 0 \prime}(\eta)+3 \gamma \bar{\chi}_{1}^{(0) \prime}(\eta)\right\} .
$$

The solution of this equation will be regular at $\eta=0$ if and only if $\mathbf{A}(0)=1$. In this case Eq. (4.27) is simply the equation satisfied by the regular part $P(\eta)$ of the singular solution 
$\bar{\chi}_{2}{ }^{0}(\eta)$ of the reduced equation. Hence, $\mathrm{G}(\eta)$ will be of the form

$$
G(\eta)=P(\eta)+C \bar{\chi}_{1}{ }^{0}(\eta)
$$

where $P(\eta)$ is given by (4.15) and $C$ is an arbitrary constant. For the present problem, we want the re-expansion of (4.21) for negative $\eta$ to be $\bar{\chi}_{2}{ }^{0}(\eta)+O\left(\epsilon^{3}\right)$. This condition requires we choose

$$
C=\log \epsilon+\frac{1}{3}(2 \psi(1)-\log 3) .
$$

Eq. (4.21) will be uniformly valid for bounded, non-positive $\eta$. The second Scorer function $G i(x)$ must be used to obtain uniform approximations to $\chi_{2}$ for non-negative $\eta$.

The dominant and recessive solutions. Consider first $\chi_{3}(\eta)$, which is exponentially small for $\eta>0$. The uniform expansion for this solution is of the form

$$
\chi_{3}(\eta)=\mathbf{a}(\eta) A i(\zeta, 1)+\epsilon^{2} \mathbf{b}(\eta) A i(\zeta, 0)+\epsilon \mathbf{c}(\eta) A i(\zeta,-1)+O\left(\epsilon^{3}\right) .
$$

Unlike Eq. (4.21) for $\chi_{2}$, the expansion (4.30) is valid for negative and positive $\eta(y)$ : [provided $y<1$ ].

Substituting (4.30) into Eq. (4.5) shows that a, b, and c satisfy the same differential equations as $\mathbf{A}, \mathbf{B}$, and $\mathbf{C}$. Hence

$$
\mathrm{a}(\eta)=\mathrm{a}(0) \bar{\chi}_{1} \cdot 0(\eta)
$$

and

$$
\mathbf{a}(\eta)+\eta \mathbf{c}(\eta)=\mathbf{a}(0) \eta^{\prime}{ }^{3 \cdot 2} .
$$

Both the constant $\mathrm{a}(0)$ and the coefficient $\mathrm{b}(\eta)$ may now be determined by requirng that the re-expansion of (4.30) for positive $\eta$ away from the turning point is identical to the WKBJ approximation $\bar{\chi}_{3}(\eta)$ in (4.17). This condition gives

$$
\mathrm{a}(0)=1
$$

and

$$
\mathbf{b}(\eta)=\eta^{-1} \mathbf{c}(\eta)+\eta^{-3 / 2} \eta^{-1 / 2}\left\{G_{1}(\eta)-\frac{41}{48} \eta^{-3 / 2}\right\} .
$$

Further, as $\mathbf{A}(0)=\mathbf{a}(0)=1$, we also have $\mathbf{B}(\eta)=\mathbf{b}(\eta)$.

Consider now $\chi_{4}(\eta)$, which is exponentially large for $\eta>0$. The uniform expansion for this solution is of the form

$$
\chi_{4}(\eta)=\mathbf{a}(\eta) B i(\zeta, 1)+\epsilon^{2} \mathbf{b}(\eta) B i(\zeta, 0)+\epsilon \mathbf{c}(\eta) B i(\zeta,-1)+O\left(\epsilon^{3}\right) .
$$

Slowly varying portions of all uniform expansions may thus be expressed in terms of three known quantities: the regular solution $\bar{\chi}_{1}^{1{ }^{\prime}}(\eta)$ of the reduced equation, the regular part $P(\eta)$ of the singular solution of the reduced equation, and the coefficient $G_{1}(\eta)$ of $\epsilon^{3 / 2}$ in the Poincaré series portion of the WKBJ approximations.

The characteristic equation. To write the characteristic equation in terms of $\eta$, let

$$
B_{2}=\frac{d^{2}}{d \eta^{2}}+\gamma \frac{d}{d \eta}, \quad B_{3}=\frac{d^{3}}{d \eta^{3}}+\left[\gamma^{\prime}+\gamma^{2}\right] \frac{d}{d \eta},
$$

and 


$$
B(X, Y)=B_{3} X(0) B_{2} Y(0)-B_{2} X(0) B_{3} Y(0) .
$$

Further, let $W(X, Y)$ denote the Wronskian of $X(\eta)$ and $Y(\eta)$ evaluated at the second boundary point $\eta_{0}$. The exact characteristic equation is then

$$
\begin{aligned}
\Delta(\alpha, \epsilon) & =B\left(\chi_{1}, \chi_{2}\right) W\left(\chi_{3}, \chi_{4}\right)-B\left(\chi_{1}, \chi_{3}\right) W\left(\chi_{2}, \chi_{4}\right) \\
& +B\left(\chi_{1}, \chi_{4}\right) W\left(\chi_{2}, \chi_{3}\right)+B\left(\chi_{2}, \chi_{3}\right) W\left(\chi_{1}, \chi_{4}\right) \\
& -B\left(\chi_{2}, \chi_{4}\right) W\left(\chi_{1}, \chi_{3}\right)+B\left(\chi_{3}, \chi_{4}\right) W\left(\chi_{1}, \chi_{2}\right) \\
& =0 .
\end{aligned}
$$

The uniform approximations $(4.18,21,30,35)$ may now be used to approximate both $B(X$, $Y)$ and $W(X, Y)$ in $\Delta$.

Consider first the $B(X, Y)$. The uniform approximations to all solutions except $\chi_{1}(\eta)$ involve $\zeta$ and $d / d \eta=\epsilon^{-1} d / d \zeta$. Hence, by $(4.36,37)$ it would appear that in (4.38) those $B$ 's involving $\chi_{1}$ should be $O\left(\epsilon^{-3}\right)$ while those $B$ 's not involving $\chi_{1}$ should be $O\left(\epsilon^{-5}\right)$. However, this is not the case, as the coefficient of the lowest-order term in the uniform approximation to both $\chi_{3}{ }^{\prime \prime \prime}$ and $\chi_{4}^{\prime \prime \prime}$ (but not $\left.\chi_{2}^{\prime \prime \prime}\right)$ vanishes at $\eta=0$. Hence, only $B\left(\chi_{2}, \chi_{3}\right)$ and $B\left(\chi_{2}\right.$, $\left.\chi_{4}\right)$ are $O\left(\epsilon^{-5}\right)$. Both $B\left(\chi_{3}, \chi_{4}\right)$ and $B\left(\chi_{1}, \chi_{2}\right)$ are $O\left(\epsilon^{-3}\right)$, while $B\left(\chi_{1}, \chi_{3}\right)$ and $B\left(\chi_{1}, \chi_{4}\right)$ are only $O\left(\epsilon^{-2}\right)$.

Approximations to the three Wronskians $W\left(\chi_{1}, Y\right)$ in $\Delta$ which involve $\chi_{1}$ do not contain products of rapidly varying quantities. Rather, the Airy or Scorer functions are multiplied only by the slowly varying coefficients and $\bar{\chi}_{1}{ }^{\circ 1}\left(\eta_{0}\right)$ and $\bar{\chi}_{1}{ }^{10 .}\left(\eta_{0}\right)$. This, however, is not the case in approximations to the three Wronskians in $\Delta$ which do not involve $\chi_{1}$. For example, approximations to $W\left(\chi_{3}, \chi_{4}\right)$ contain the products $A i\left(\zeta_{0}, p\right) B i\left(\zeta_{0}, q\right)$ with $p, q$ $=0, \pm 1$ and $p \neq q$. Fortunately, the Wronskian relationships $(4.8-12)$ can be used to eliminate such products. For example, the approximation to $W\left(\chi_{3}, \chi_{4}\right)$ may be written in the form

$$
\begin{gathered}
W\left(\chi_{3}, \chi_{4}\right)=\epsilon^{-1} \mathbf{a}\left(\eta_{0}\right) \eta_{0}{ }^{\prime-3 / 2} W[A i(\zeta, 1), B i(\zeta, 1)]\left(\zeta_{0}\right)-\eta_{0}{ }^{-3 / 2} \mathbf{c}\left(\eta_{0}\right) W[A i(\zeta, 0), B i(\zeta, 0)]\left(\zeta_{0}\right) \\
+\epsilon\left[\mathbf{a}(\mathbf{b}+\mathbf{c})-\mathbf{a}^{\prime} \mathbf{c}\right]\left(\eta_{0}\right) \frac{d}{d \zeta} W[A i(\zeta, 1), B i(\zeta, 1)]\left(\zeta_{0}\right)+O\left(\epsilon^{2}\right)
\end{gathered}
$$

Using $(4.9,10)$ and $(4.31)$ now gives

$$
\begin{gathered}
W\left(\chi_{3}, \chi_{4}\right)=\epsilon^{-1} \eta_{0}{ }^{-3 / 2} \bar{\chi}_{1}^{10}\left(\eta_{0}\right)\left[\pi^{-1} H i\left(\zeta_{0}, 0\right)-B i\left(\zeta_{0}, 0\right)\right] \\
-\pi^{-1} \eta_{0}{ }^{\prime-3 / 2} \mathbf{c}\left(\eta_{0}\right) \\
+\epsilon\left[\mathbf{a}\left(\mathbf{b}+\mathbf{c}^{\prime}\right)-\mathbf{a}^{\prime} \mathbf{c}\right]\left(\eta_{0}\right)\left[\pi^{-1} H i\left(\zeta_{0},-1\right)-B i\left(\zeta_{0},-1\right)\right]+O\left(\epsilon^{2}\right) .
\end{gathered}
$$

Similar simplifications are possible for $W\left(\chi_{2}, \chi_{3}\right)$ and $W\left(\chi_{2}, \chi_{4}\right)$. However, as these terms multiply $B\left(\chi_{1}, \chi_{3}\right)$ and $B\left(\chi_{1}, \chi_{4}\right)$ in $\Delta$, they are not required to approximate $\Delta$ with relative error $O\left(\epsilon^{3}\right)$.

The approximations to the $B$ 's and $W$ 's may now be used to obtain uniform approximations to $\Delta$. As

$$
A i(0)=3^{-1 / 2} B i(0)=3^{-2 / 3} / \Gamma\left(\frac{2}{3}\right), \quad A i^{\prime}(0)=-3^{-1 / 2} B i^{\prime}(0)=-3^{-1 / 3} / \Gamma\left(\frac{1}{3}\right),
$$

the relationships between the Bessel and Airy functions 


$$
\begin{aligned}
J_{ \pm 1 / 3}(\hat{x}) & =\frac{1}{2}\left(\frac{3}{x}\right)^{1 / 2}\left[3^{1 / 2} A i(-x) \mp B i(-x)\right], \\
J_{ \pm 2 / 3}(\hat{x}) & =\frac{3^{1 / 2}}{2 x}\left[ \pm 3^{1 / 2} A i^{\prime}(-x)+B i^{\prime}(-x)\right], \\
\hat{x} & =\frac{2}{3} x^{3 / 2}
\end{aligned}
$$

may be used to further simplify the resulting expression. With relative error $O\left(\epsilon^{3}\right)$, we now obtain the surprisingly simple form

$$
\begin{aligned}
\frac{3^{1 / 2}}{2} \epsilon^{13 / 2} \Delta(\alpha, \epsilon)= & -\left|\eta_{0}\right|^{1 / 2} \eta_{0}{ }^{-3 / 2} \bar{\chi}_{1}^{{ }^{10}}\left(\eta_{0}\right)\left\{A i^{\prime}(0) J_{-1 / 3}\left(\xi_{0}\right)+\epsilon\left(\lambda-\frac{1}{10}\right) \operatorname{Ai}(0) J_{1 / 3}\left(\xi_{0}\right)\right. \\
& \left.+\epsilon^{5 / 2}\left[\left(\lambda^{2}-\frac{\lambda}{5}+\frac{59}{525}\right) B i\left(\zeta_{0}\right)-\left(\frac{3 \lambda^{2}}{2}-\frac{6 \lambda}{5}+\frac{59}{350}\right) \operatorname{Hi}\left(\zeta_{0}\right)\right]\right\} \\
& +\epsilon^{32} \eta_{0}\left[\left(\mathrm{~b}+\mathrm{c}^{\prime}\right) \bar{\chi}_{1}{ }^{0}-\mathbf{c} \bar{\chi}_{1}{ }^{0 \prime \prime}\right]\left(\eta_{0}\right) A i^{\prime}(0) J_{2 / 3}\left(\xi_{0}\right)+O\left(\epsilon^{3}\right)
\end{aligned}
$$

where

$$
\xi_{0}=\frac{2}{3}\left|\zeta_{0}\right|^{3 / 2}
$$

Approximations to the eigenvalues $\lambda_{n}(\alpha, \epsilon)$ for $\alpha>1$ may be obtained by setting (4.42) to zero. Although $\alpha$ is considered fixed, the relevant expansion for $\lambda_{n}$ in powers of $\epsilon$ now depends on the size of $\alpha$. If $\alpha$ is $O(1)$, (4.42) implies $\lambda_{n}=\lambda_{n}{ }^{10}+\epsilon^{3 / 2} \lambda_{n}{ }^{11}+\cdots$. In this case, $\xi_{0}$ and $\zeta_{0}$ are large. If, on the other hand, $\alpha=O\left(\epsilon^{-1}\right)$, then both $\zeta_{0}$ and $\xi_{0}$ are $O(1)$. In this case, $\eta_{0}$ lies inside the critical layer about $\eta=0$ and the relevant expansion for $\lambda_{n}$ is $\lambda_{n}$ $=\lambda_{n}{ }^{\prime 0}+\epsilon \lambda_{n}{ }^{11}+\cdots$. Our primary concern in the present work is obtaining $\lambda_{n}{ }^{0}$.

The Bessel function $J_{-1 / 3}\left(\xi_{0}\right)$ in (4.42) has zeros on the positive real axis. For fixed $\alpha$, let $\epsilon_{c}$ denote a value of $\epsilon$ for which $J_{-1 / 3}\left(\xi_{0}\left(\alpha, \epsilon_{c}\right)\right)=0$. Two cases must now be considered:

a) $\epsilon \neq \epsilon_{c}$. By (4.42), expanding $\lambda_{n}$ and setting $\Delta=0$ implies that with $\lambda_{n}=\lambda_{n}{ }^{0}$, $\bar{\chi}_{1}{ }^{0}\left(\eta_{0}\right)=0$, or, from $(4.16)$,

$$
P_{\nu_{n}^{\prime} 0}\left(\frac{\alpha}{\alpha-1}\right)=0 .
$$

We now have

$$
\lambda_{n}^{0:}=\nu_{n}^{0}\left(\nu_{n}^{0}+1\right) / 2 .
$$

Hence, $\lambda_{n}{ }^{10}$ will come from a zero in degree of the Legendre function.

b) $\epsilon=\epsilon_{c}$. In this case $J_{-1 / 3}\left(\xi_{0}\right)$ vanishes but $J_{1 / 3}\left(\xi_{0}\right)$ will be non-zero. Hence, $\Delta=0$ implies either $\bar{\chi}_{1}{ }^{01}\left(\eta_{0}\right)=0$ as before or $\lambda_{n}{ }^{0 !}=\frac{1}{10}$. The latter choice would lead to eigenvalues which are discontinuous at the points $\epsilon_{c}$ and whose first approximations are independent of $n$. Hence, this choice must be rejected, and we again have Eq. (4.44) for $\nu_{n}{ }^{0}$.

If $\nu$ is real, $P_{\nu}(x)$ can vanish only for $x$ in the real interval $(-1,1)$. In the present case with $\alpha>1, \alpha /(\alpha-1)>1$. Hence, zeros in degree of $P_{\nu}(\alpha /(\alpha-1))$ must be complex. However, the real part of the zeros now is uniquely fixed by the fact that $\alpha /(\alpha-1)$ is real. Hille, in a 1918 paper [3], has shown that the zeros must be of the form

$$
\nu_{n}{ }^{|0|}=-\frac{1}{2}+i \beta_{n} \quad(n=0,1,2, \cdots)
$$


with all $\beta_{n}$ positive. The Legendre functions $P_{\nu}$ are thus conical functions. Hille's results further indicate that $\beta_{0}$ increases with $\alpha$. The eigenvalues for $\alpha>1$ are now $\lambda_{n}{ }^{(0)}=-\frac{1}{2}\left(\beta_{n}{ }^{2}\right.$ $\left.+\frac{1}{4}\right)$ which are real but negative. Hence, the frequencies $\omega_{n}=\Omega \lambda_{n}{ }^{1 / 2}$ will be purely imaginary, and the small transverse vibrations will grow in time without oscillations.

A comment on physical interpretations. In a physical situation involving a rotating elastic rod, the geometric off-clamping parameter $\alpha$ is known and fixed. However, the rod cannot be constrained to vibrate in a single plane with a specified angle $\theta$. Instead, small physical disturbances will involve vibrations in planes with a spectrum of angles, $\theta_{0} \leq \theta \leq$ $\theta_{1}$. If $\alpha$ is non-zero, the present results show that the amplitude of vibrations in planes with angles $\theta_{0} \leq \theta<\theta_{c}(\alpha)$ will grow with time. Hence, as $\theta_{0}$ is usually zero, rapidly rotating rods with even small off-clamping will be unstable. The present linear theory also predicts that instability will first be observed in the plane of rotation, as this plane has the maximal growth rate for unstable vibrations.

5. Buckling in the transverse plane for $\alpha>\frac{1}{2}$. As a complement to any discussion of time-varying phenomena such as the vibration of a beam, it is natural to include a description of time-independent solutions. Clearly, the existence of these stationary states (buckled modes) provides the explanation for the appearance of eigenvalues corresponding to unbounded amplitudes of vibration. This is essentially due to the fact that we are examining a sophisticated version of the problem of the vibrations of a beam subjected to an axial load [2]. In our case, not only is the loading a function of position, but also the plane of vibration (and buckling) must be explicitly considered.

For the present problem there are buckled states for every angle $\theta$ when $\alpha>\frac{1}{2}$. The case $\theta=0$ was examined by Nachman [11] and we shall provide an analysis for the case $\theta=$ $\pi / 2$.

An examination of the equation for the transverse vibrations (2.1) shows that for $\theta=$ $\pi / 2$ time-independent states are solutions of the equation

$$
\frac{d^{4} u}{d x^{4}}-\mu^{2} \frac{d}{d x}\left[(1-x)\left\{\frac{1}{2}(1+x)-\alpha\right\} \frac{d u}{d x}\right]=0
$$

with associated boundary conditions

$$
u(0)=u^{\prime}(0)=u^{\prime \prime}(1)=u^{\prime \prime \prime}(1)=0,
$$

where

$$
\mu^{2}=\rho A \Omega^{2} L^{4} / E I
$$

and, for convenience, the interval of interest is now $[0,1]$.

Eq. (5.1) may be integrated from $x$ to 1. Letting $v(x)=u^{\prime}(x)$ then gives

$$
\begin{gathered}
v^{\prime \prime}-\mu^{2}(1-x)\left[\frac{1}{2}(1+x)-\alpha\right] v=0, \\
v(0)=v^{\prime}(1)=0 .
\end{gathered}
$$

For general $\alpha>\frac{1}{2}$, the solution $v(x, \alpha)$ of (5.4) will involve parabolic cylinder functions leading to a complicated eigenvalue relationship. However, in the important case $\alpha=1$, considerable simplification is possible. A solution $v(x, 1)$ such that $v^{\prime}(1,1)=0$ is

$$
v(x, 1)=(1-x)^{1 / 2} J_{-1 / 4}\left[2^{-3 / 2} \mu(1-x)^{2}\right]
$$

where $J_{-1 / 4}$ is the Bessel function of order $-\frac{1}{4}$. If $j_{-1 / 4, n}$ is the $n$th positive zero of $J_{-1 / 4}(z)$ 
and $\mu_{n}=\mu_{n}(\alpha, \theta)$, then $v(0,1)=0$ gives for $n=0,1,2, \cdots$ that

$$
\mu_{n}(1, \pi / 2)=2^{3 / 2} j_{-1 / 4, n+1} .
$$

In particular, $\mu_{0}(1, \pi / 2)=5.6746$ and $\mu_{1}(1, \pi / 2)=14.4899$.

Buckling is to be expected on physical grounds when $\alpha>\frac{1}{2}$ as every point of the rod is under compression due to the rotation. However, as discussed in the next section, the possibility of buckling cannot automatically be dismissed if compressive forces are absent.

6. Non-transverse buckling for $0<\alpha<\frac{1}{2}$. When $0 \leq \alpha<\frac{1}{2}$, every point of the rod is under tension as a result of the rotation. Transverse vibrations are stable, and there are no static buckled states in the transverse plane $\theta=\pi / 2$. However, the present work on unstable vibration predicts that despite the tension forces, static buckling is possible in non-transverse planes when $\alpha<\frac{1}{2}$. Consider, for example, a plane with fixed angle $\theta, 0 \leq \theta$ $<\pi / 2$. The asymptotic results of Sec. 3 show that the first vibration eigenvalue $\lambda_{0}(\alpha, \theta)$ becomes negative for $\alpha$ in the range $\alpha_{c}(\theta)<\alpha<\frac{1}{2}$. However, the second vibration eigenvalue $\lambda_{1}(\alpha, \theta)$ remains positive for all $\alpha<\frac{1}{2}$. These factors suggest the existence of exactly one buckled mode. In particular, they predict that for each $0 \leq \theta<\pi / 2$, the boundary-value problem

$$
\begin{gathered}
\frac{d^{4} u}{d x^{4}}-\mu^{2}\left\{\frac{d}{d x}\left\{(1-x)\left[\frac{1}{2}(1+x)-\alpha\right] \frac{d u}{d x}\right\}+\left(\cos ^{2} \theta\right) u\right\}=0 \\
u(0)=u^{\prime}(0)=u^{\prime \prime}(1)=u^{\prime \prime \prime}(1)=0 \\
\alpha_{c}(\theta)<\alpha<\frac{1}{2}
\end{gathered}
$$

which governs the static buckling of the rod should have exactly one positive eigenvalue.

Unfortunately, applying asymptotic methods to (6.1) yields only the information that the eigenvalue $\mu^{2}$ cannot be both large and positive. However, approximations to $\mu^{2}$ may be obtained using a Ritz-Galerkin technique [8]. Consider, for example, static in-plane buckling of the rod. In accordance with the asymptotic prediction, for $\theta=0$ it was found that the boundary-value problem (6.1) has exactly one positive eigenvalue $\mu^{2}=0(1)$ when $0.05<\alpha<\frac{1}{2}$. Thus, despite the tension, the rotating rod will have a static in-plane buckled state for even small off-clamping.

\section{REFERENCES}

[1] W. E. Boyce, R. C. DiPrima, and G. H. Handleman, Vibrations of rotating beams of constant section, in Second U.S. Nat. Congress Appl. Mech., 165-173 (1956)

[2] D. J. Gorman, Free vibration analysis of beams and shafts, Wiley, New York, 1975

[3] E. Hille, Some problems concerning spherical harmonics, Ark. Mat., Astron., Fys. 13, 1-76 (1918)

[4] E. W. Hobson, The theory of spherical and ellipsoidal harmonics, Chelsea Publishing Co., New York, 1965

[5] W. D. Lakin, On the differential equation of a rapidly rotating slender rod, Quart. Appl. Math. 32, 11-27 (1974)

[6] W. D. Lakin, Vibrations of a rotating flexible rod clamped off the axis of rotation, J. Eng. Math. 10, 313-321 (1976)

[7] W. D. Lakin and B. S. Ng, A fourth order eigenvalue problem with a turning point at the boundary, Quart. J. Mech. Appl. Math. 28, 107-121 (1975)

[8] R. A. Mathon, private communication

[9] J. C. P. Miller, On the choice of standard solutions for a homogeneous linear differential equation of the second order. Quart. J. Mech. Appl. Math. 3, 225-235 (1950)

[10] N. Mostaghel and I. Tadjbachsh, Buckling of rotating rods and plates, Int. J. Mech. Sci. 15, 429-434 (1973) 
[11] A. Nachman, The buckling of rotating rods, J. Appl. Mech. 42, 222-224 (1975)

[12] Jahnke-Emde, Tables of higher functions, McGraw-Hill, New York, 1960

[13] W. H. Reid, Composite approximations to the solutions of the Orr-Sommerfeld equation. Studies Appl. Math. 51, 341-368 (1972)

[14] W. H. Reid, Uniform asymptotic approximations to the solutions of the Orr-Sommerfeld equation, part 2: the general theory. Studies Appl. Math. 53, 217-224 (1974)

[15] Y. Luke, Integrals of Bessel functions, McGraw-Hill, New York, 1962

[16] F. W. J. Olver, Asymptotics and special functions, Academic Press, New York, 1974 